\title{
Shallow Water Stony Corals (Scleractinia, Milleporidae, and Stylasteridae) from Utila and Cayos Cochinos, Honduras
}

\author{
Patrick Scaps ${ }^{1}$ and James Saunders ${ }^{2}$ \\ ${ }^{1}$ Laboratoire de Biologie Animale, Université des Sciences et Technologies de Lille, 59655 Villeneuve d'Ascq Cédex, France \\ ${ }^{2}$ Coral View Research Centre, Sandy Bay, Utila, Honduras \\ Correspondence should be addressed to Patrick Scaps, patrick.scaps@univ-lille1.fr
}

Received 29 January 2011; Accepted 12 March 2011

Academic Editors: K. Cheng and M. Klautau

Copyright ( 2011 P. Scaps and J. Saunders. This is an open access article distributed under the Creative Commons Attribution License, which permits unrestricted use, distribution, and reproduction in any medium, provided the original work is properly cited.

\begin{abstract}
The coral reefs of Utila and Cayos Cochinos located in the western Caribbean region (Honduras) were studied in Summer 2010 in order to acquire information concerning their richness in stony corals (Scleractinia, Milleporidae, and Stylasteridae) down to $18 \mathrm{~m}$ depth. Ten sites in each location were visually surveyed. Fourty-six species and 12 forms of zooxanthellate scleractinian corals belonging to 21 genera were observed at Utila and 44 species and 12 forms belonging to 20 genera at Cayos Cochinos. At each site, 3 species of milleporids and one species of Stylaster (S. roseus) were also observed. Four forms of zooxanthellate scleractinian corals were new records from Honduras. Up to now, 53 species of scleractinian corals have been identified within the whole Honduran Caribbean coast. They belong to 24 genera. This number represents approximately $93 \%$ of all scleractinian coral species reported for the Caribbean.
\end{abstract}

\section{Introduction}

The Caribbean coastline of Honduras, Central America, represents the southern end of the Mesoamerican Barrier Reef System, although its marine resources are less extensive and studied than nearby Belize and Mexico [1]. However, the coastal zone contains mainland reef formations and a highly developed small island reef system which can be divided into three groups, the Bay Islands and Cayos Cochinos archipelago, the Mosquita cays and banks, and the small Swan Islands. The Bay Islands group has a number of smaller cays but is dominated by three major islands: Utila, Roatán, and Guanaja. These islands which rise up from deep water are relatively long and thin and oriented in a nearly east-west direction. Although small, they are surrounded by fringing reefs.

Data concerning zooxanthellate scleractinian diversity in Honduras are very scarce. Up to now, only a limited number of authors studied the scleractinian diversity in Honduras. Tortora and Keith [2] presented a checklist of 15 species that they collected in the Swan Islands (some $150 \mathrm{~km}$ northeast of the mainland) during the summer 1974. Fenner [3] found 35 zooxanthellate and 6 azooxanthellate species of scleractinian corals plus 3 species of hydrocorals off the northwestern Roatán Island in April 1987 to a depth of $30 \mathrm{~m}$. More recently, Guzman [4] determined the diversity of the coral fauna in all habitats down to $35 \mathrm{~m}$ depth at the Reserva Biologica Cayos Cochinos from surveys conducted from August 1994 to June 1996. He reported 53 species of zooxanthellate scleractinian species plus three Millepora.

The objectives of this study were to obtain data about scleractinian diversity at Utila and to update the checklist of Guzman [4] from Cayos Cochinos in order to complete the systematic list of zooxanthellate scleractinian coral fauna of Honduras and to compare scleractinian diversity in different areas in Honduras and with other localities of the western Caribbean.

\section{Material and Methods}

2.1. Study Area. The study area included Utila island, one of the Bay Islands, and Cayos Cochinos archipelago. Utila, which has 13 cays, is the southernmost island in the Bay Islands group and is located $29 \mathrm{~km}$ off the coast of Honduras 


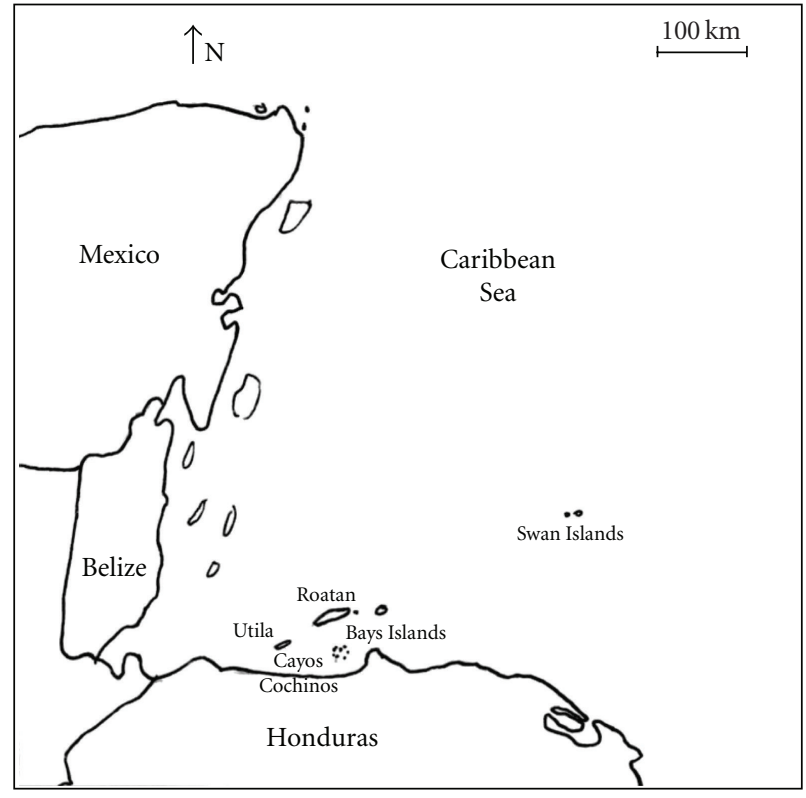

(a)

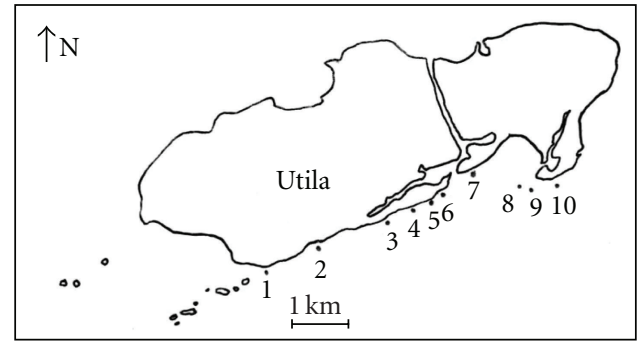

(b)

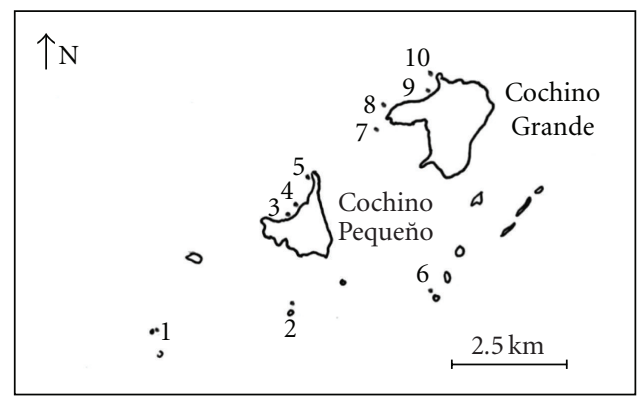

(c)

Figure 1: (a) Map of Caribbean showing location of Utila and Cayos Cochinos. (b) Utila dive sites: (1) Sting Ray Point; (2) Jack Neil Beach; (3) Little Bight; (4) Pretty Bush; (5) Silver Gardens; (6) Black Coral Wall; (7) House Reef; (8) Lighthouse Reef; (9) Ron's Wreck; (10) Moon Hole. (c) Cayos cochinos dive sites: (1) Timon; (2) Paloma: (3) Jena’s Cove: (4) Punto del Alex; (5) Menor West; (6) El Avion; (7) Aggressor Mooring; (8) Pelican Point; (9) Lions Head; and (10) Lions Paw.

(Figure 1(a)). Cayos Cochinos is located on the continental shelf approximately $16 \mathrm{~km}$ off the coast of mainland Honduras and $46 \mathrm{~km}$ south-east of Utila in the western Caribbean Sea (Figure 1(a)). A deep trough (>400 m) separates Cayos Cochinos and Utila. Fringing coral reefs line the coasts of Utila, providing protection from storms. The Cayos Cochinos are comprised of two small hilly islands (Cochino Pequeňo and Cochino Grande) and 13 tiny coral cays. Tropical forests cover both Cochino Pequeňo and Cochino Grande, while most of the cays are sandbars. Reefs at Cayos Cochinos are a combination of fringing reefs around the cays and barrier reef mounts.

Though the reef of Utila and Cayos Cochinos share many similarities, they have important differences. Historically, Utila has been bathed in clear water ( $>30 \mathrm{~m}$ visibility), which is a result of relatively strong oceanic currents that sweep past the island. However, during the past decade, poor land-use practices and development have resulted in increased runoff and sediment deposition on the reefs. Cayos Cochinos, on the other hand, is located on the shallow continental shelf and is permanently influenced by runoff from mainland rivers that result in salinity, temperature, turbidity, and water-quality fluctuations. Land clearing and deforestation on the Honduran mainland has probably accelerated sediment loading, nutrient content, and frequency of flood events that eventually impact the marine environment around Cayos Cochinos, and to a minor extent, other Bay Islands. The Cayos Cochinos archipelago has been shown to be within reach of local sediment plumes following the severe impacts of Huricane Mitch in 1998 [6].
In 1997, legislation was passed declaring most of the Bay Islands as a marine park with varying levels of restrictions on resource use. However, although the whole perimeter of Roatan and Guannaja and parts of Utila were included, enforcement is limited and the forestry department, which is responsible for protected areas, has virtually no capacity on the islands [1]. On the contrary, the reefs of Cayos Cochinos were established as a Biological Reserve in November 1993 and banned all commercial harvest (nonartisanal) of marine life within a $489 \mathrm{~km}^{2}$ area [7].

2.2. Methods. Observations were carried out in the field by diving during the summer of 2010. 10 stations were surveyed at Utila and Cayos Cochinos, respectively. Stations were chosen on the basis of accessibility and a need for a representative spread around the islands. In Utila, diving was mostly done on the south side of the island (Figure 1(b)). Diving was done on the north-west sides of Cochino Pequeňo and Cochino Grande; a few dives also took place on barely emergent reefs elsewhere (Figure 1(c)). Maximum depth $(18 \mathrm{~m})$ and maximum dive times (45 minutes) were in compliance with the standards of Operation Wallacea. The basic method consisted of underwater observations. The name of each species identified underwater was marked on a plastic sheet on which species names were preprinted. Dives consisted generally of a slow ascent along the reef in a zigzag path to the shallowest points. Sample areas of all habitats encountered were surveyed, including sandy areas, walls, overhangs, slopes, and shallow reefs. Areas typically hosting few or no corals, such as seagrass beds and mangroves, were 


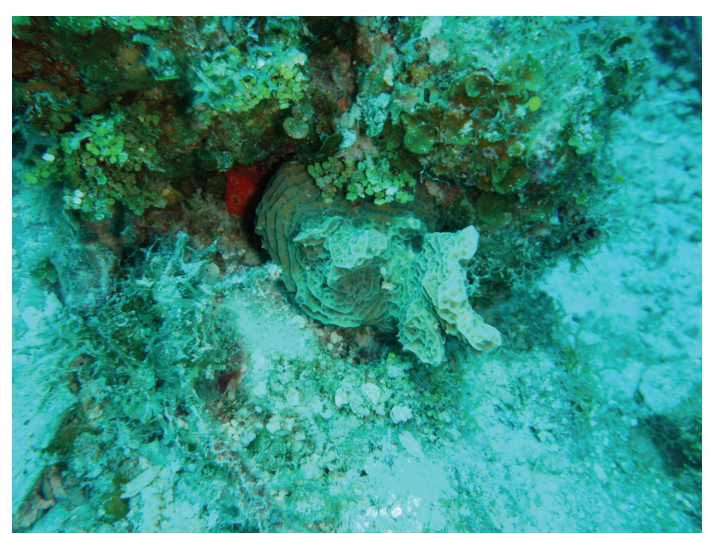

(a)

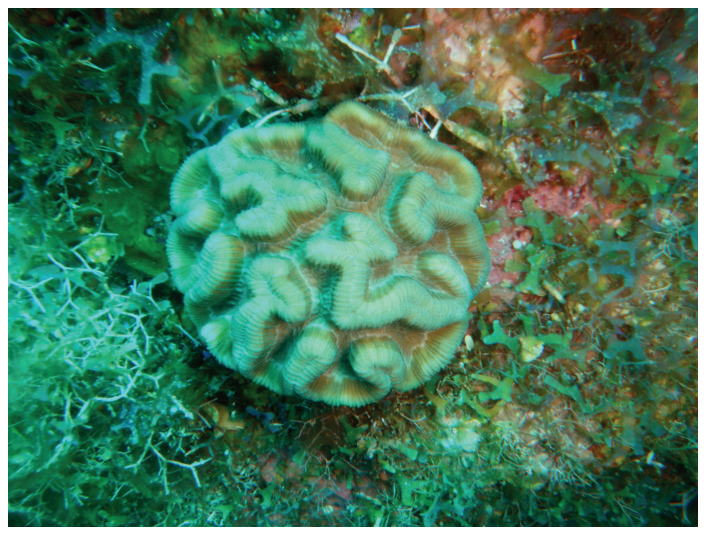

(c)

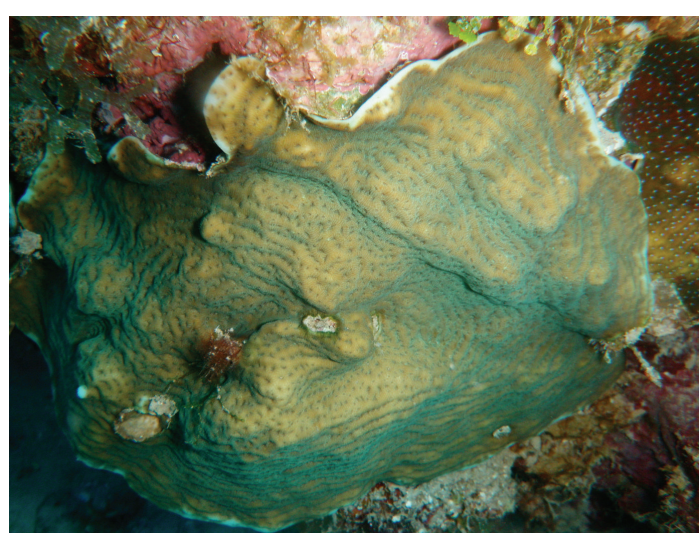

(b)

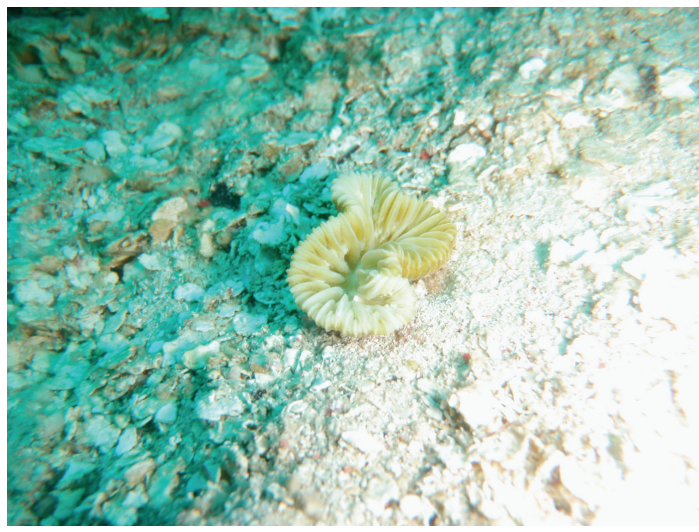

(d)

Figure 2: New forms of zooxanthellate scleractinian corals for Honduras. (a) Agaricia agaricites forma carinata (Wells, 1973). (b) Agaricia fragilis forma contracta (Wells, 1973). (c) Manicia areolata forma mayori (Wells, 1943). (d) Meandrina meandrites forma danai (Linnaeus, $1758)$.

not surveyed. All the species and forms encountered during this study were photographed in the field by the first author.

The stony corals recorded included the zooxanthellate scleractinian corals and a small number of zooxanthellate and azooxanthellate nonscleractinian corals (e.g., Millepora and Stylaster "fire coral" and "lace coral"). All produce calcium carbonate skeletons that contribute to reef building to some degree. Stony coral species were identified visually according to Almy and Carrion-Torres [8], Wells [9], Cairns [10], Zlatarski and Estelella [11], de Weerdt [12], Fenner [5], and Human and Deloach [13]. Due to the different criteria used by the authors to define the dates and names of the describers of the species, we used the most recent publications. Collections were not made due to the conservation nature of the Operation Wallacea program. The coral taxonomy used was that of Wells and Lang [14], with modifications from Cairns [10], Van Moorsel [15], de Weerdt [12], Zlatarski [16], Weil [17], Fenner [5, 18], Weil and Knowlton [19], Vermeij et al. [20], and Locke et al. [21].

Relative abundances are listed as abundant where they are found at each dive site and predominant by numbers or coverage of substratum; common are found at each dive site but are not predominant species; uncommon are not found at each dive site but observed regularly; those listed as rare were not found at each dive site and observed irregularly. Our estimates of species richness of Honduras were complemented with data from other sources [2-4].

\section{Results}

Table 1 lists the stony corals (Scleractinia, Milleporidae, and Stylasteridae) that were observed at Utila and Cayos Cochinos in July 2010 in the shallower $(<18 \mathrm{~m})$ zones of the reefs. Coral species richness appeared quite similar at the two locations although some differences were notable. At Utila 46, species and 12 forms of zooxanthellate scleractinian corals belonging to 21 genera were observed compared to 44 species and 12 forms belonging to 20 genera at Cayos Cochinos. At each site, 4 species of hydrozoans (three Millepora and Stylaster roseus) were also observed. The agaricid Agaricia undata and the mussid Isophyllastrea rigida were not observed at Cayos Cochinos while they were classified as rare at Utila. An average of 37.1 species of zooxanthellate scleractinian corals was found per site at Utila and 33.4 at Cayos Cochinos. 
TABle 1: Stony corals (Scleractinia, Milleporidae, and Stylasteridae) of Utila and Cayos Cochinos, Honduras. Abundant: species observed at each dive site and predominant by numbers or coverage of substratum; common: species observed at each dive site but not a predominant species; uncommon: species not observed at each dive but observed regularly; rare: species not observed at each dive and observed irregularly.

\begin{tabular}{|c|c|c|}
\hline Species & Utila & Cayos cochinos \\
\hline \multicolumn{3}{|l|}{ CLASS HYDROZOA (Owen, 1843) } \\
\hline \multicolumn{3}{|l|}{ ORDER ATHECATAE (Hincks, 1868) } \\
\hline \multicolumn{3}{|l|}{ FAMILLY MILLEPORIDAE (Fleming, 1901) } \\
\hline (1) Millepora alcicornis (Linnaeus, 1758) & Common & Common \\
\hline (2) Millepora complanata (Lamarck, 1816) & Common & Common \\
\hline (3) Millepora squarosa (Lamarck, 1816) & Uncommon & Uncommon \\
\hline \multicolumn{3}{|l|}{ FAMILLY STYLASTERIDAE (Gray, 1847) } \\
\hline (4) Stylaster roseus* (Pallas, 1766) & Uncommon & Uncommon \\
\hline \multicolumn{3}{|l|}{ CLASS ANTHOZOA (Ehrtenberg, 1834) } \\
\hline \multicolumn{3}{|l|}{ ORDER SCLERACTINIA (Bourne, 1900) } \\
\hline \multicolumn{3}{|l|}{ SUBORDER ASTROCOENIINA (Vaughan \& Wells, 1943) } \\
\hline \multicolumn{3}{|l|}{ FAMILLY ASTROCOENIIDAE (Koby, 1890) } \\
\hline (1) Stephanocoenia intersepta (Lamarck, 1816) ${ }^{\mathrm{a}}$ & Common & Common \\
\hline \multicolumn{3}{|l|}{ FAMILLY POCILLOPORIDAE (Gray, 1842) } \\
\hline (2) Madracis auretenra (Loche, Weil \& Coates, 2007) ${ }^{\mathrm{b}}$ & Abundant & Common \\
\hline (3) Madracis decactis (Lyman, 1859) & Common & Common \\
\hline (4) Madracis formosa (Wells, 1973) & Rare & Rare \\
\hline (5) Madracis pharensis forma luciphila (Wells, 1973) & Uncommon & Uncommon \\
\hline Madracis pharensis forma pharensis* (Heller, 1868) & Uncommon & Uncommon \\
\hline \multicolumn{3}{|l|}{ FAMILLY ACROPORIDAE (Verrill, 1902) } \\
\hline (6) Acropora cervicornis (Lamarck, 1816) & Uncommon & Uncommon \\
\hline (7) Acropora palmata (Lamarck, 1816) & Uncommon & Uncommon \\
\hline (8) Acropora prolifera (Lamarck, 1816) & Rare & Rare \\
\hline \multicolumn{3}{|l|}{ SUBORDER FUNGIINA (Verrill, 1865) } \\
\hline \multicolumn{3}{|l|}{ SUPERFAMILLY AGARICIICAE (Gray, 1847) } \\
\hline \multicolumn{3}{|l|}{ FAMILLY AGARICIIDAE (Gray, 1847) } \\
\hline (9) Agaricia agaricites forma agaricites (Linnaeus, 1758) & Abundant & Abundant \\
\hline Agaricia agaricites forma carinata (Wells, 1973) & Common & Common \\
\hline Agaricia agaricites forma danai (Milne Edwards \& Haime, 1860) & Common & Common \\
\hline Agaricia agaricites forma purpurea (Lesueur, 1821) & Rare & Rare \\
\hline (10) Agaricia fragilis forma contracta (Wells, 1973) & Rare & Rare \\
\hline Agaricia fragilis forma fragilis (Dana, 1846) & Common & Common \\
\hline (11) Agaricia grahamae (Wells, 1973) & Rare & Rare \\
\hline (12) Agaricia humilis (Verrill, 1901) & Common & Common \\
\hline (13) Agaricia lamarcki (Milne-Edwards \& Haime, 1851) & Common & Uncommon \\
\hline (14) Agaricia tenuifolia (Dana, 1846) & Common & Common \\
\hline (15) Agaricia undata (Ellis \& Solander, 1786) & Rare & Not observed \\
\hline (16) Leptoseris cucullata (Ellis \& Solander, 1786) & Common & Common \\
\hline \multicolumn{3}{|l|}{ FAMILLY SIDERASTREIDAE (Vaughan \& Wells, 1943) } \\
\hline (17) Siderastrea radians (Pallas, 1766) & Common & Common \\
\hline (18) Siderastrea siderea (Ellis \& Solander, 1786) & Abundant & Abundant \\
\hline \multicolumn{3}{|l|}{ SUPERFAMILLY PORITICAE (Gray, 1842) } \\
\hline \multicolumn{3}{|l|}{ FAMILLY PORITIDAE (Gray, 1842) } \\
\hline (19) Porites astreoides (Lamarck, 1816) & Abundant & Abundant \\
\hline (20) Porites colonensis (Zlatarski, 1990) & Uncommon & Rare \\
\hline (21) Porites divaricata (Lesueur, 1821) & Uncommon & Uncommon \\
\hline (22) Porites furcata (Lamarck, 1816) & Abundant & Abundant \\
\hline (23) Porites porites (Pallas, 1766) & Abundant & Abundant \\
\hline
\end{tabular}


TABle 1: Continued.

\begin{tabular}{|c|c|c|}
\hline Species & Utila & Cayos cochinos \\
\hline \multicolumn{3}{|l|}{ SUBORDER FAVIINA (Vaughan \& Wells, 1943) } \\
\hline \multicolumn{3}{|l|}{ FAMILLY FAVIIDAE (Gregory, 1900) } \\
\hline \multicolumn{3}{|l|}{ SUBFAMILLY FAVIINAE (Gregory, 1900) } \\
\hline (24) Colpophyllia natans forma breviserialis (Milne-Edwards \& Haime, 1849) & Rare & Rare \\
\hline Colpophyllia natans forma natans (Houttuyn, 1772) & Common & Common \\
\hline (25) Diploria clivosa (Ellis \& Solander, 1786) & Uncommon & Uncommon \\
\hline (26) Diploria labyrinthiformis (Linnaeus, 1758) & Common & Common \\
\hline (27) Diploria strigosa (Dana, 1846) & Common & Common \\
\hline (28) Favia fragum (Esper, 1795) & Uncommon & Uncommon \\
\hline (29) Manicia areolata forma areolata (Linnaeus, 1758) & Uncommon & Common \\
\hline Manicia areolata forma mayori (Wells, 1936) & Rare & Rare \\
\hline \multicolumn{3}{|l|}{ SUBFAMILLY MONTASTREINAE (Vaughan \& Wells, 1943) } \\
\hline (30) Montastrea annularis (Ellis \& Solander, 1786) & Abundant & Abundant \\
\hline (31) Montastrea cavernosa (Linnaeus, 1766) & Common & Common \\
\hline (32) Montastrea faveolata (Ellis \& Solander, 1786) & Abundant & Abundant \\
\hline (33) Montastrea franksi (Gregory, 1895) & Abundant & Abundant \\
\hline \multicolumn{3}{|l|}{ FAMILLY MEANDRINIDAE (Gray, 1847) } \\
\hline \multicolumn{3}{|l|}{ SUBFAMILLY MEANDRININAE (Gray, 1847) } \\
\hline (34) Meandrina meandrites forma brasiliensis (Milne-Edwards \& Haime, 1848) & Rare & Rare \\
\hline Meandrina meandrites forma danai (Linnaeus, 1758) & Common & Common \\
\hline Meandrina meandrites forma meandrites (Linnaeus, 1758) & Common & Common \\
\hline Meandrina meandrites forma memorialis (Wells, 1973) & Uncommon & Uncommon \\
\hline \multicolumn{3}{|l|}{ SUBFAMILLY DICHOCOENIINAE (Vaughan \& Wells, 1943) } \\
\hline (35) Dendrogyra cylindrus (Ehrenberg, 1834) & Uncommon & Rare \\
\hline (36) Dichoecoenia stokesi forma stellaris (Milne-Edwards \& Haime, 1849) & Rare & Rare \\
\hline Dichoecoenia stokesi forma stokesi (Milne-Edwards \& Haime, 1848) & Uncommon & Uncommon \\
\hline \multicolumn{3}{|l|}{ FAMILLY MUSSIDAE (Ortman, 1890) } \\
\hline (37) Isophyllastrea rigida (Dana, 1846) & Rare & Not observed \\
\hline (38) Isophyllia sinuosa forma multiflora (Verrill, 1902) & Uncommon & Uncommon \\
\hline Isophyllia sinuosa forma sinuosa (Ellis \& Solander, 1786) & Uncommon & Uncommon \\
\hline (39) Mussa angulosa (Pallas, 1766) & Uncommon & Uncommon \\
\hline (40) Mycetophyllia aliciae (Wells, 1973) & Common & Common \\
\hline (41) Mycetophyllia danaana (Milne-Edwards \& Haime, 1849) & Common & Common \\
\hline (42) Mycetophyllia ferox (Wells, 1973) & Common & Common \\
\hline (43) Mycetophyllia lamarckiana (Milne-Edwards \& Haime, 1848) & Common & Common \\
\hline (44) Scolymia cubensis (Milne-Edwards \& Haime, 1849) & Uncommon & Uncommon \\
\hline (45) Scolymia lacera (Pallas, 1766) & Uncommon & Uncommon \\
\hline \multicolumn{3}{|l|}{ SUBORDER CARYOPHYLLINA (Vaughan \& Wells, 1943) } \\
\hline \multicolumn{3}{|l|}{ FAMILLY CARYOPHYLLIIDAE (Gray, 1847) } \\
\hline (46) Eusmilia fastigiata forma fastigiata (Pallas, 1766) & Common & Common \\
\hline
\end{tabular}

${ }^{a}$ Previously reported as Stephanocoenia michelinii (Milne Edwards and Haime, 1848).

${ }^{b}$ Previously reported as Madracis mirabilis (Duchassaing and Michelotti, 1860).

*Ahermatypic.

Abundances of stony corals appeared quite similar at the two locations (Table 1). Most of the reefs were dominated by Montastrea annularis, M. faveolata, M. franksi, Porites astreoides, P. porites, P. furcata, Siderea sidereal, and Agaricia agaricites. Subtle differences in species occurrence were observed in Agaricia lamarcki commonly found at Utila, yet uncommon at Cayos Cochinos. Alternatively, Manicia areolata forma mayori was commonly found at Cayos Cochinos but uncommon at Utila. Madracis auretenra was abundant at Utila and commonly seen at Cayos Cochinos, and Porites colonensis and Dendrogyra cylindrus were uncommon at Utila and rare at Cayos Cochinos.

During this study, we did not encounter some species (Madracis senaria, Agaricia undata, Porites branneri, Cladocora arbuscula, Solenastrea bournoni, Solenastrea hyades, Oculina diffusa, Isophyllia rigida, and Mycetophyllia reesi) and 
TABLe 2: Stony corals (Scleractinia, Milleporidae, and Stylasteridae) species presence-absence list for Honduras.

\begin{tabular}{l}
\hline Species \\
CLASS HYDROZOA (Owen, 1843) \\
ORDER ATHECATAE (Hincks, 1868) \\
FAMILLY MILLEPORIDAE (Fleming, 1901) \\
(1) Millepora alcicornis (Linnaeus, 1758) \\
(2) Millepora complanata (Lamarck, 1816) \\
(3) Millepora squarosa (Lamarck, 1816) \\
ORDER STYLASTERINA (Hickson \& England, 1905) \\
FAMILLY STYLASTERIDAE (Gray, 1847) \\
(4) Stylaster roseus* (Pallas, 1766) \\
CLASS ANTHOZOA (Ehrtenberg, 1834) \\
ORDER SCLERACTINIA (Bourne, 1900) \\
SUBORDER ASTROCOENIINA (Vaughan \& Wells, 1943 \\
FAMILLY ASTROCOENIIDAE (Koby, 1890) \\
(1) Stephanocoenia intersepta (Lamarck, 1816) \\
FAMILLY POCILLOPORIDAE (Gray, 1842) \\
(2) Madracis auretenra (Loche, Weil \& Coates, 2007) \\
(3) Madracis decactis (Lyman, 1859) \\
(4) Madracis formosa (Wells, 1973) \\
(5) Madracis pharensis forma luciphila (Wells, 1973) \\
Madracis pharensis forma pharensis* (Heller, 1868)
\end{tabular}

\section{1}

2

3

4

CLASS HYDROZOA (Owen, 1843)

ORDER ATHECATAE (Hincks, 1868)

FAMILLY MILLEPORIDAE (Fleming, 1901)

(2) Millepora complanata (Lamarck, 1816)

(3) Millepora squarosa (Lamarck, 1816

ORDER STYLASTERINA (Hickson \& England, 1905)

FAMILLY STYLASTERIDAE (Gray, 1847)

(4) Stylaster roseus* (Pallas, 1766)

CLASS ANTHOZOA (Ehrtenberg, 1834)

ORDER SCLERACTINIA (Bourne, 1900)

Wells, 1943)

(1) Stephanocoenia intersepta (Lamarck, 1816)

FAMILLY POCILLOPORIDAE (Gray, 1842)

(2) Madracis auretenra (Loche, Weil \& Coates, 2007) ${ }^{\mathrm{b}}$

(3) Madracis decactis (Lyman, 1859)

4) Madracis formosa (Wells, 1973)

6) Madracis senaria (Wells, 1973)

FAMILLY ACROPORIDAE (Verrill, 1902)

(7) Acropora cervicornis (Lamarck, 1816)

(8) Acropora palmata (Lamarck, 1816)

(9) Acropora prolifera (Lamarck, 1816)

SUBORDER FUNGIINA (Verrill, 1865)

SUPERFAMILLY AGARICIICAE (Gray, 1847)

FAMILLY AGARICIIDAE (Gray, 1847)

(10) Agaricia agaricites forma agaricites (Linnaeus, 1758)

Agaricia agaricites forma carinata (Wells, 1973)

Agaricia agaricites forma danai (Milne Edwards \& Haime, 1860)

Agaricia agaricites forma purpurea (Lesueur, 1821)

(11) Agaricia fragilis forma contracta (Wells, 1973)

Agaricia fragilis forma fragilis (Dana, 1846)

(12) Agaricia grahamae (Wells, 1973)

(13) Agaricia humilis (Verrill, 1901)

(14) Agaricia lamarcki (Milne-Edwards \& Haime, 1851)

(15) Agaricia tenuifolia (Dana, 1846)

(16) Agaricia undata (Ellis \& Solander, 1786)

(17) Leptoseris cucullata (Ellis \& Solander, 1786)

FAMILLY SIDERASTREIDAE (Vaughan \& Wells, 1943)

(18) Siderastrea radians (Pallas, 1766)

(19) Siderastrea siderea (Ellis \& Solander, 1786)

SUPERFAMILLY PORITICAE (Gray, 1842)

FAMILLY PORITIDAE (Gray, 1842)

(20) Porites astreoides (Lamarck, 1816)

(21) Porites branneri (Rathbun, 1887)

(22) Porites colonensis (Zlatarski, 1990)

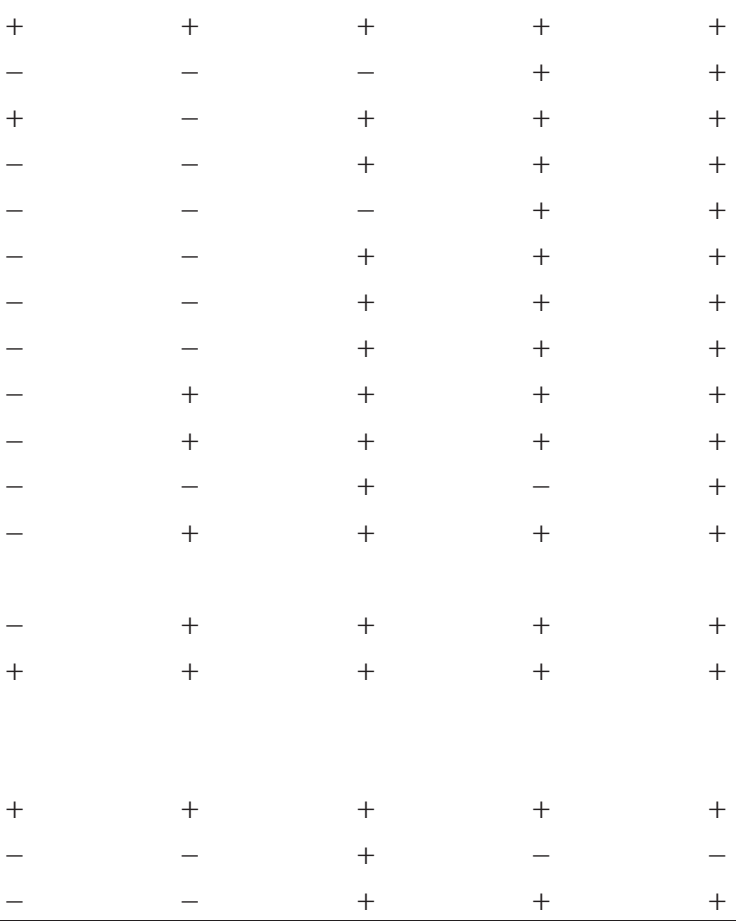


TABle 2: Continued.

\begin{tabular}{|c|c|c|c|c|c|}
\hline Species & 1 & 2 & 3 & 4 & 5 \\
\hline (23) Porites divaricata (Lesueur, 1821) & - & + & + & + & + \\
\hline (24) Porites furcata (Lamarck, 1816) & + & + & + & + & + \\
\hline (25) Porites porites (Pallas, 1766) & + & + & + & + & + \\
\hline \multicolumn{6}{|l|}{ SUBORDER FAVIINA (Vaughan \& Wells, 1943) } \\
\hline \multicolumn{6}{|l|}{ FAMILLY FAVIIDAE (Gregory, 1900) } \\
\hline \multicolumn{6}{|l|}{ SUBFAMILLY FAVIINAE (Gregory, 1900 ) } \\
\hline (26) Colpophyllia natans forma breviserialis (Milne-Edwards \& Haime, 1849) & + & + & + & + & + \\
\hline Colpophyllia natans forma natans (Houttuyn, 1772) & - & + & + & + & + \\
\hline (27) Diploria clivosa (Ellis \& Solander, 1786) & + & + & + & + & + \\
\hline (28) Diploria labyrinthiformis (Linnaeus, 1758) & - & + & + & + & + \\
\hline (29) Diploria strigosa (Dana, 1846) & - & + & + & + & + \\
\hline (30) Favia fragum (Esper, 1795) & + & + & + & + & + \\
\hline (31) Manicia areolata forma areolata (Linnaeus, 1758) & - & + & + & + & + \\
\hline Manicia areolata forma mayori (Wells, 1936) & - & - & - & + & + \\
\hline \multicolumn{6}{|l|}{ SUBFAMILLY MONTASTREINAE (Vaughan \& Wells, 1943) } \\
\hline (32) Cladocora arbuscula (Lesueur, 1821) & - & - & + & - & - \\
\hline (33) Montastrea annularis (Ellis \& Solander, 1786) & + & + & + & + & + \\
\hline (34) Montastrea cavernosa (Linnaeus, 1766) & + & + & + & + & + \\
\hline (35) Montastrea faveolata (Ellis \& Solander, 1786) & - & - & + & + & + \\
\hline (36) Montastrea franksi (Gregory, 1895) & - & - & + & + & + \\
\hline (37) Solenastrea bournoni (Milne Edwards \& Haime, 1849) & - & - & + & - & - \\
\hline (38) Solenastrea hyades (Dana, 1846) & - & - & + & - & - \\
\hline \multicolumn{6}{|l|}{ FAMILLY OCULINIDAE (Gray, 1847) } \\
\hline (39) Oculina diffusa (Lamarck, 1816) & - & - & + & - & - \\
\hline \multicolumn{6}{|l|}{ FAMILLY MEANDRINIDAE (Gray, 1847) } \\
\hline \multicolumn{6}{|l|}{ SUBFAMILLY MEANDRININAE (Gray, 1847) } \\
\hline $\begin{array}{l}\text { (40) Meandrina meandrites forma brasiliensis } \\
\text { (Milne-Edwards \& Haime, 1848) }\end{array}$ & - & - & + & + & + \\
\hline Meandrina meandrites forma danai (Linnaeus, 1758) & - & - & - & + & + \\
\hline Meandrina meandrites forma meandrites (Linnaeus, 1758) & - & + & + & + & + \\
\hline Meandrina meandrites forma memorialis (Wells, 1973) & - & - & + & + & + \\
\hline \multicolumn{6}{|l|}{ SUBFAMILLY DICHOCOENIINAE (Vaughan \& Wells, 1943) } \\
\hline (41) Dendrogyra cylindrus (Ehrenberg, 1834) & - & + & + & + & + \\
\hline $\begin{array}{l}\text { (42) Dichoecoenia stokesi forma stellaris } \\
\text { (Milne-Edwards \& Haime, 1849) }\end{array}$ & - & - & + & + & + \\
\hline $\begin{array}{l}\text { Dichoecoenia stokesi forma stokesi } \\
\text { (Milne-Edwards \& Haime, 1848) }\end{array}$ & - & + & + & + & + \\
\hline \multicolumn{6}{|l|}{ FAMILLY MUSSIDAE (Ortman, 1890) } \\
\hline (43) Isophyllastrea rigida (Dana, 1846) & + & + & + & - & + \\
\hline (44) Isophyllia sinuosa forma multiflora (Verrill, 1902) & + & + & + & + & + \\
\hline Isophyllia sinuosa forma sinuosa (Ellis \& Solander, 1786) & + & - & + & + & + \\
\hline (45) Mussa angulosa (Pallas, 1766) & - & + & + & + & + \\
\hline (46) Mycetophyllia aliciae (Wells, 1973) & - & + & + & + & + \\
\hline (47) Mycetophyllia danaana (Milne-Edwards \& Haime, 1849) & - & + & + & + & + \\
\hline (48) Mycetophyllia ferox (Wells, 1973) & + & + & + & + & + \\
\hline (49) Mycetophyllia lamarckiana (Milne-Edwards \& Haime, 1848) & - & + & + & + & + \\
\hline (50) Mycetophyllia reesi (Wells, 1973) & - & - & + & - & - \\
\hline (51) Scolymia cubensis (Milne-Edwards \& Haime, 1849) & - & + & + & + & + \\
\hline (52) Scolymia lacera (Pallas, 1766) & - & - & + & + & + \\
\hline
\end{tabular}


Table 2: Continued.

\begin{tabular}{|c|c|c|c|c|c|}
\hline Species & 1 & 2 & 3 & 4 & 5 \\
\hline \multicolumn{6}{|l|}{ SUBORDER CARYOPHYLLINA (Vaughan \& Wells, 1943) } \\
\hline \multicolumn{6}{|l|}{ FAMILLY CARYOPHYLLIIDAE (Gray, 1847) } \\
\hline (53) Eusmilia fastigiata forma fastigiata (Pallas, 1766) & - & + & + & + & + \\
\hline Eusmilia fastigiata forma flabellata (Wells, 1973) & - & - & + & - & - \\
\hline
\end{tabular}

forms (Eusmilia fastigiata forma flabellata) reported found at Cayos Cochinos by Guzman [4] (Table 2). Four forms of zooxanthellate scleractinian corals observed during this study (Agaricia agaricites forma carinata, Agaricia fragilis forma contracta, Manicia areolata forma mayori, and Meandrina meandrites forma danai) have not been reported for Honduras in the published literature [2-4] (Table 2). These forms are illustrated in Figure 2.

\section{Discussion}

The comparison of shallow water stony corals between Utila and Cayos Cochinos reveals no significant differences in important reef building species. Throughout the study area, the stony coral species richness and abundance were uniform. Two species were not found at Cayos Cochinos (Agaricia undata and Isophyllastrea rigida) but they were also rarely observed at Utila. The only differences in abundances of zooxanthellate scleractinian corals between the two sites concern Madracis auretenra, Agaricia lamarcki, Porites colonensis, and Dendrogyra cylindrus which were less common at Cayos Cochinos than at Utila and Manicia areolata forma mayori which was more common at Cayos Cochinos than at Utila. The coral reefs of Utila and Cayos Cochinos currently support diverse and abundant populations of stony corals and appear to be typical of that of many other areas in the Caribbean in that they include many of the species common elsewhere. Most of the reefs at Utila and Cayos Cochinos were dominated by Montastrea annularis, M. faveolata, M. franksi, Porites astreoides, P. porites, P. furcata, Siderea sidereal, and Agaricia agaricites. Many of these species are dominant in Caribbean reef systems.

We observed much more species of zooxanthellate scleractinian species at Utila (46 species) and Cayos Cochinos (44 species) than Tortora and Keith [2] in the Swan Islands (only 15 species) and that Fenner [3] off the northwestern Roatán (35 species) which is another island of the Bay Islands group. Nevertheless, we found less zooxanthellate scleractinian species in Cayos Cochinos than previously reported by Guzman [4] (44 versus 53). This is not surprising because the study of Guzman lasted longer (from August 1994 to June 1996) and extended to deeper areas (down to $35 \mathrm{~m}$ depth) than our study. Unfortunately, Guzman did not give information about species relative abundances. It can be assumed that the species we have not observed during this study have been rarely observed by Guzman and that these species can be considered rare in Honduras. Nevertheless, we reported for the first time four new forms of zooxanthellate scleractinian corals not observed by Guzman at Cayos Cochinos.

A complete list of stony corals species observed at Honduras is given including our own results as well as documented records published by previous authors (Table 2). This list includes various formae and ecomorphs which are considered to be separated species by some researchers. The list comprises 53 species and 13 forms of zooxanthellate scleractinian corals, 3 species of Millepora, and one species of Stylaster (S. roseus). The number of zooxanthellate scleractinian species found at Honduras is slightly higher than those reported from other western Caribbean reefs at Belize (51 species and 8 forms) and Cozumel Mexico (49 species and 8 forms) [18]. Thus, the number of zooxanthellate scleractinian corals reported indicate that the reefs of Honduras are well within the range reported for other western Caribbean reefs.

The 53 species of zooxanthellate scleractinian corals known from Honduras represent 93\% of the approximately 57 zooxanthellate species known from the Caribbean. This is consistent with the view that most zooxanthellate scleractinian corals range throughout the Caribbean [3, 18, 22, 23]. In that sense, Chiappone et al. [24] showed that the diversity of hermatypic scleractinian corals is probably similar and evenly distributed in the central and western regions of the Caribbean.

\section{Acknowledgments}

The authors would like to thank Operation Wallacea (and their partner in Honduras) for their financial contributions and logistical support in Honduras and the volunteer divers who assisted with diving. They thank also the Honduran Coral Reef Fund for providing permission to the study in Los Cayos Cochinos.

\section{References}

[1] A. R. Harborne, D. C. Afzal, and M. J. Andrews, "Honduras: Caribbean Coast," Marine Pollution Bulletin, vol. 42, no. 12, pp. 1221-1235, 2001.

[2] L. R. Tortora and D. E. Keith, "Scleractinian corals of the Swan islands, Honduras," Caribbean Journal of Science, vol. 16, pp. 65-72, 1980. 
[3] D. P. Fenner, "Some reefs and corals of Roatan (Honduras), Cayman Brac, and Little Cayman," Atoll Research Bulletin, vol. 338, pp. 1-30, 1993.

[4] H. M. Guzman, "Diversity of stony, soft and black corals (Anthozoa: Scleractinia, Gorgonacea, Hydrozoa: Milleporina) at Cayos Cochinos, Bay Islands, Honduras," Revista de Biología Tropical, vol. 46, no. 4, supplement, pp. 75-80, 1998.

[5] D. P. Fenner, "Species distinctions among several Caribbean stony corals," Bulletin of Marine Science, vol. 53, no. 3, pp. 1099-1116, 1993.

[6] S. Andrefouet, P. J. Mumby, M. McField, C. Hu, and F. E. Muller-Karger, "Revisiting coral reef connectivity," Coral Reefs, vol. 21, no. 1, pp. 43-48, 2002.

[7] K. Clifton and L. M. Clifton, "A survey of fishes from various coral reef habitats within the Cayos Cochinos Marine Reserve, Honduras," Revista de Biología Tropical, vol. 46, no. 4, supplement, pp. 109-124, 1998.

[8] C. Almy and C. Carrion-Torres, "Shallow water stony corals of Puerto Rico," Caribbean Journal of Science, vol. 3, pp. 133-162, 1963.

[9] J. W. Wells, "New and old scleractinian corals from Jamaica," Bulletin of Marine Science, vol. 23, pp. 16-54, 1973.

[10] S. D. Cairns, "Stony corals (Cnidaria: Hydrozoa, Scleractinia) of Carrie Bow Cay, Belize," Smithsonian Contribution to the Marine Science, vol. 12, pp. 271-302, 1982.

[11] V. N. Zlatarski and N. Estalella, Les Scléractiniaires de Cuba, Académie Bulgare des Sciences, Sofia, Bulgaria, 1982.

[12] W. H. de Weerdt, "Taxonomic characters in Caribbean Millepora species (Hydrozoa, Coelenterata)," Bijdragen tot de Dierkunde, vol. 54, pp. 543-262, 1984.

[13] P. Human and N. Deloach, Reef Coral Identification, New World Publications, Jachsonville, Fla, USA, 2002.

[14] J. W. Wells and J. C. Lang, "Systematic list of Jamaican shallowwater Sleractinia," Bulletin of Marine Science, vol. 23, pp. 55$58,1973$.

[15] G. W. N. M. Van Moorsel, "Reproductive strategies in two closely related stony corals (Agaricia, Scleractinia)," Marine Ecology Progress Series, vol. 13, pp. 272-283, 1983.

[16] V. N. Zlatarski, "Porites colonensis, new species of stony coral (Anthozoa: Scleractinia) off the Caribbean coast of Panama," Proceedings of the Biological Society of Washington, vol. 103, pp. 257-264, 1990.

[17] E. Weil, "Genetic and morphological variation in Caribbean and eastern Pacific Porites (Anthozoa, Scleractinia). Preliminary results," in Proceedings of the 7th International Coral Reef Symposium, vol. 2, pp. 643-656, 1992.

[18] D. Fenner, "New observations on the stony coral (Scleractinia, Milleporidae, and Stylasteridae) species of Belize (Central America) and Cozumel (Mexico)," Bulletin of Marine Science, vol. 64, no. 1, pp. 143-154, 1999.

[19] E. Weil and N. Knowlton, "A multi-character analysis of the Caribbean coral Montastraea annularis (Ellis and Solander, 1786) and its two sibling species, M. faveolata (Ellis and Solander, 1786) and M. franksi (Gregory, 1895)," Bulletin of Marine Science, vol. 55, no. 1, pp. 151-175, 1994.

[20] M. J. A. Vermeij, D. E. Fiekmann, and R. P. M. Bak, "A new sepcies of Scleractinian Coral (Cnidaria, Abthozoa) Madracis carmabi N. Sp. from the Caribbean," Bulletin of Marine Science, vol. 73, no. 3, pp. 679-684, 2003.

[21] J. M. Locke, E. Weil, and K. A. Coates, "A newly documented species of Madracis (Scleractinia: Pocilloporidae) from the Caribbean," Proceedings of the Biological Society of Washington, vol. 120, no. 2, pp. 214-226, 2007.
[22] W. D. Liddel and S. L. Ohlhorst, "Comparison of Western Atlantic coral reef communities," in Proceedings of the 6th International Coral Reef Symposium, vol. 3, pp. 281-286, 1988.

[23] W. H. de Weerdt, "Discontinuous distribution of the tropical West Atlantic hydrocoral Millepora squarrosa," Beaufortia, vol. 41, pp. 195-203, 1990.

[24] M. Chiappone, K. M. Sullivan, and C. Lott, "Hermatypic scleractinian corals of the Southeastern Bahamas: a comparison to western Atlantic reef systems," Caribbean Journal of Science, vol. 32, no. 1, pp. 1-13, 1996. 

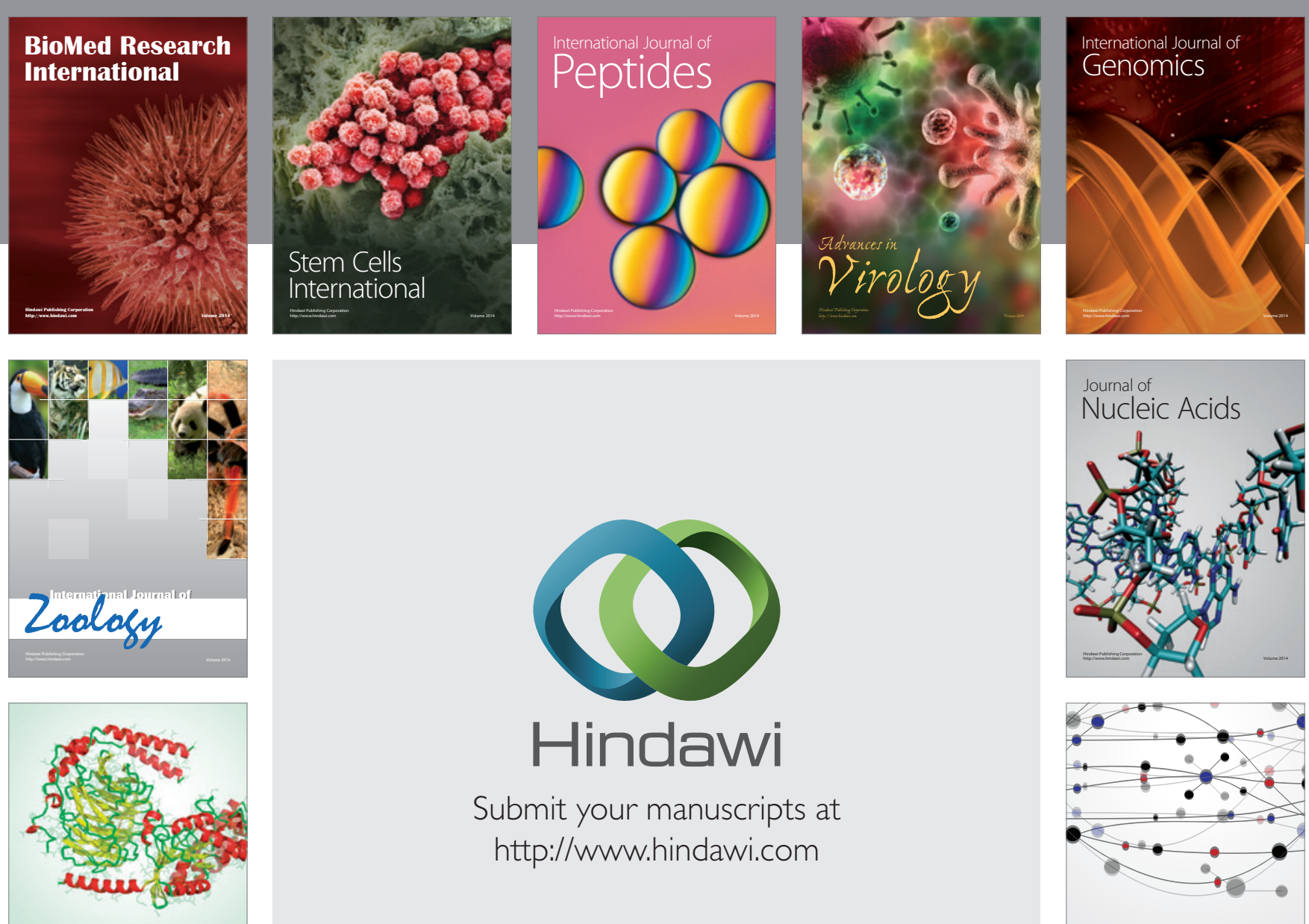

Submit your manuscripts at

http://www.hindawi.com

Signal ${ }^{\text {Jumal }}$ Transduction
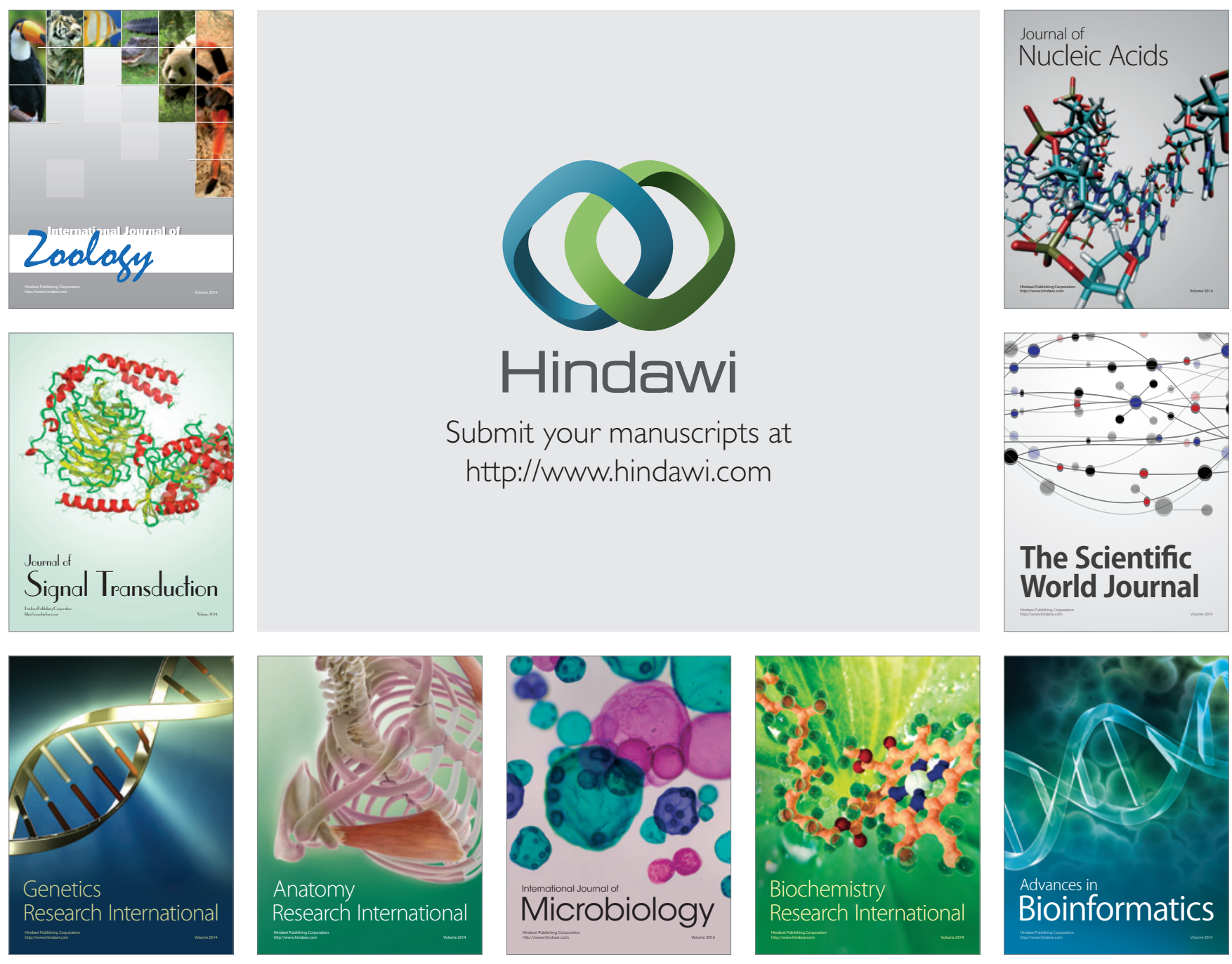

The Scientific World Journal
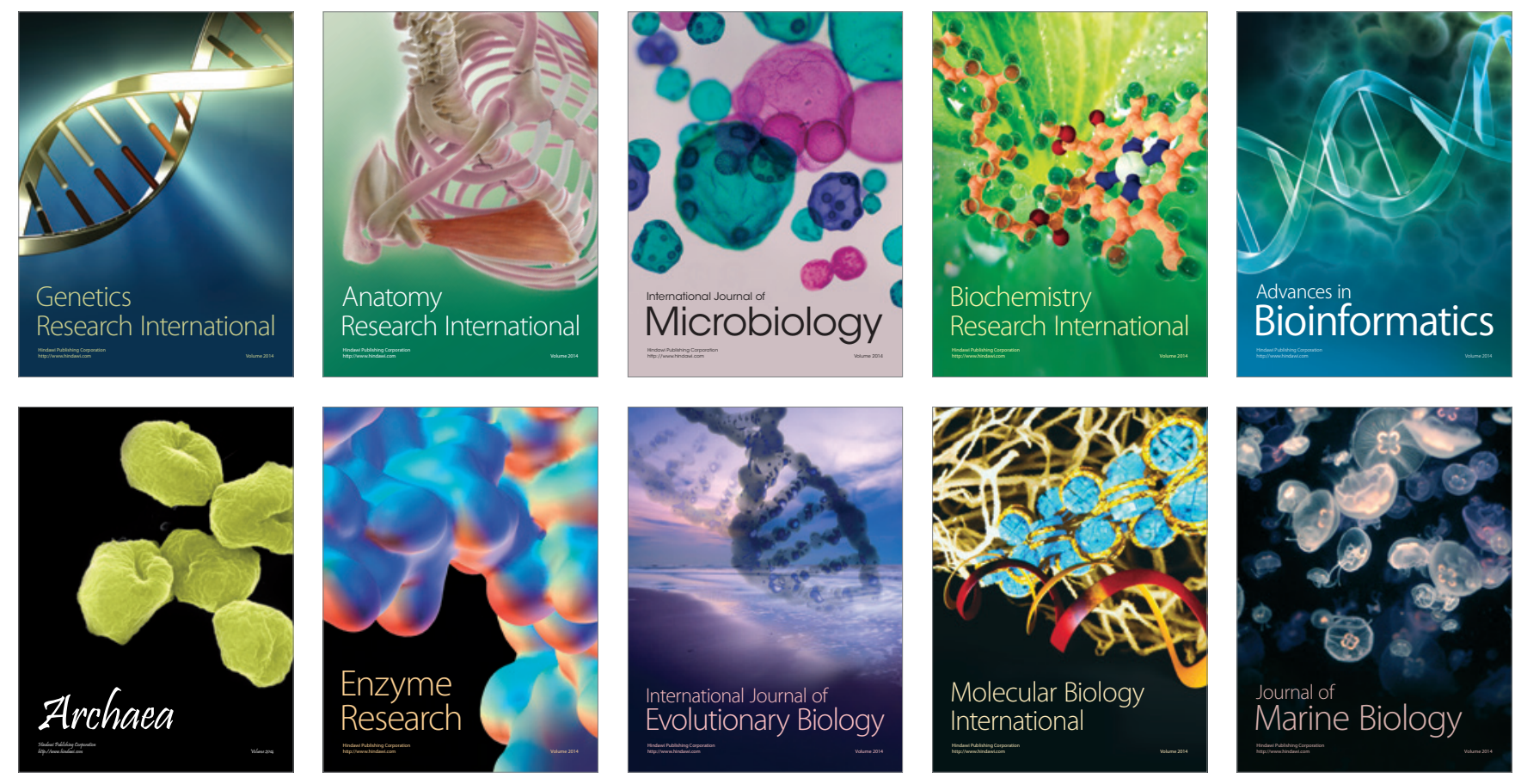